\title{
Stratigraphic studies of Ganymede's tectonic activity in the bright terrain: results from the Byblus Sulcus and Harpagia Sulcus regions
}

\author{
Namitha Rose Baby ${ }^{1}$, Katrin Stephan ${ }^{1}$, Roland Wagner ${ }^{1}$, Katrin Krohn ${ }^{1}$, and Nico Schmedemann ${ }^{2}$ \\ ${ }^{1}$ Institute of Planetary Research, DLR, Berlin, Germany \\ ${ }^{2}$ University of Muenster, Muenster, Germany
}

The Jovian satellite Ganymede experienced a pronounced period of tectonic resurfacing forming the extended bright or light terrain the so-called Sulci, which cover about 2/3 of Ganymede's surface (Pappalardo et al., 2004 and references therein). It crosscuts the older dark terrain of the so-called regions by long swaths of subparallel grooves or separates the different regions by a complex network of tens to kilometer wide polygons/cells (Patterson et al., 2010, Collins et al., 2013), with each cell being characterized by a different density and orientation of the structural grooves. Previous studies indicated that different tectonic styles are apparent reaching from roughly evenly spaced grooves oriented in a single dominant direction to smooth surface areas with only faint or undetectable grooves at decameter to kilometer resolution (Collins et al., 1998, Patterson et al., 2010) with cryovolcanic resurfacing playing a possible role in the formation of the latter.

In order to better understand the formation of the bright terrain and its possible interaction with a subsurface ocean its investigation has been made one of the top goals of the upcoming JUICE mission (Grasset et al., 2013). For the purpose of preparing for the JUICE mission and maximizing its science return, we re-investigate the available Voyager ISS and Galileo SSI data set. Our focus lies on the characterization of the bright terrain, its contact to the dark terrain, the definition and characterization of the tectonic subunits/cells and their stratigraphic relationship to each other. The work is supported by the estimation of the surface ages from crater frequency distributions and compositional information derived from Galileo NIMS data. The goal is to study the local formation process and to identify any changes in tectonic style through time, but also to compare the formation process of the bright terrain across Ganymede's entire surface in order to reveal any global/regional differences in the past tectonic activity, and to characterize possible differences and similarities of the bright terrain at different locations on the body.

Among the studied areas are a portion of Byblus Sulcus ( $40^{\circ} \mathrm{N} / 160^{\circ} \mathrm{E}$ ) and of Harpagia Sulcus $\left(\sim 16^{\circ} \mathrm{S} / 50^{\circ} \mathrm{E}\right)$ located in the northern portion of the anti-Jovian hemisphere and in the southern portion of the sub-Jovian hemisphere, respectively. Byblus Sulcus is a $30 \mathrm{~km}$ broad and NNW-SSE oriented band reaching from Philus Sulcus into the dark terrain of Marius Regio, where it meets the east-west trending grooved lane named Akitu Sulcus, which is truncated by the grooved and smooth 
terrain of Byblus Sulcus and thus is relatively older. Whereas the ancient neighboring dark terrain is already highly furrowed, Byblus Sulcus is mainly characterized by grooved terrain (Igf and $I g c$ ) and smaller areas of smooth terrain (Is) on its northern border to the dark terrain of Marius Regio. Byblus Sulcus is superimposed by small two dark floor craters with a dark inner and bright outer halo of ejecta material representing the youngest features in this region.

The imaged portion of Harpagia Sulcus covers a 20-km broad region of bright or light material units including the light grooved terrain $(I g)$, a smooth-subdued terrain in the middle $(I s)$, the lineated terrains (II) on either sides of the smooth-subdued terrain and a region of undivided terrain (undiv) on the southern side of the Sulcus. The smooth-subdued terrain accounts for a comparably large number of craters as compared to other terrains and is being crosscut by the lineated terrains on either sides and the light grooved terrain on the eastern and southern sides, evidenced by the presence of sharp boundaries separating them. So, the smooth-subdued terrain is supposed to be older than other terrains. The light grooved terrain is supposed to be much younger terrain since it crosscuts the lineated terrain and accounts for the smaller number of craters. Lineated terrains to be intermediate in age between light grooved terrain and smooth-subdued terrain. Nevertheless, crater density frequency indicates a narrow timescale.

While the crater frequency areas of measurement of the dark terrain units in Byblus Sulcus are clearly higher than those from bright terrain, inferring a higher age, the bright units in both the Byblus and Harpagia Sulcus show crater frequencies more or less identical within measurement uncertainties. Whether this is a general feature of Ganymede's bright terrain, or specific to these two selected regions, will be a major issue in our ongoing studies. Also, bright grooved and smooth units cannot be separated very well in terms of crater frequencies in both regions. Since the existing cratering chronology models by Neukum et al. (1998) and Zahnle et al. (2003) are highly uncertain, it is difficult to infer if Ganymede was tectonically active only over a short period of time or over a much longer period, as implied by low impact rates in the Jovian system (e.g., Zahnle et al., 2003). 
Ganymede: Harpagia (HS) and Byblus Sulcus (BS)

Bright subdued (smooth) terrain (HS: blue; BS: green)

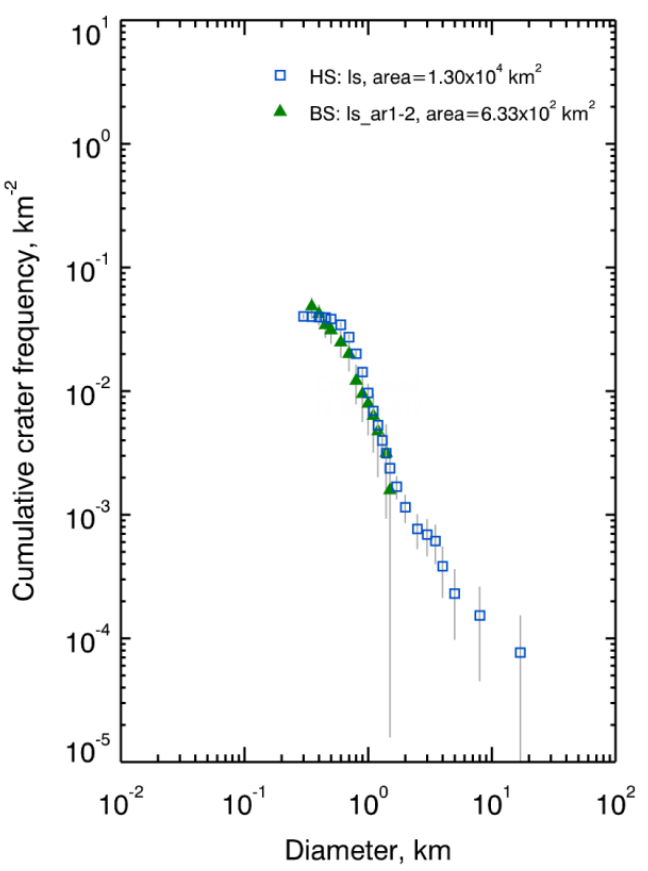

Ganymede: Harpagia (HS) and Byblus Sulcus (BS)

Bright grooved and lineated terrain (HS: blue; BS: green)

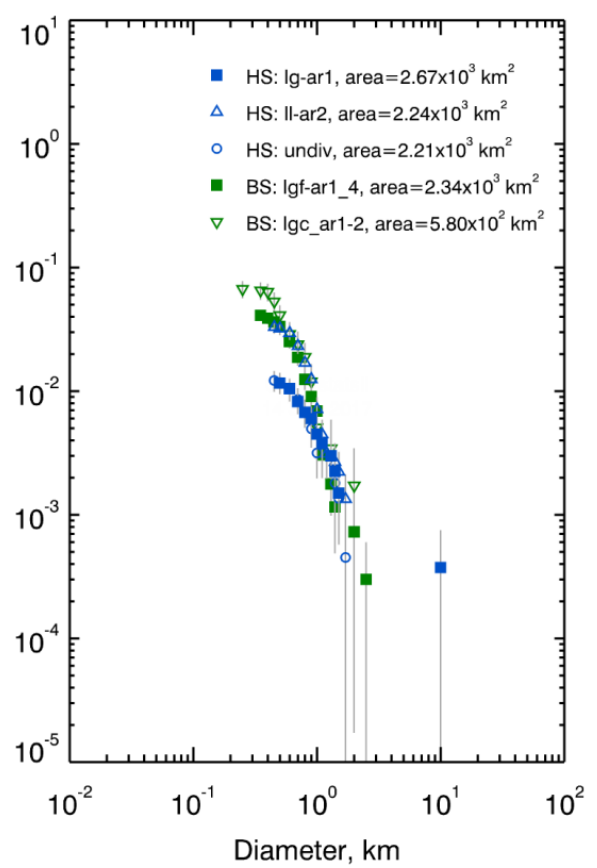

Figure 1: Crater size frequency distributions for the studied geological units in Byblus (BS) and Harpagia Sulcus (HS). (Measured units: a) HS - bright smooth terrain (Is), bright grooved (Ig), lineated (II) and undivided terrain (undiv) and BS bright grooved terrain is subdivided into areas at the flanks (Igf) and in the central portions $(I g c)$ of Byblus Sulcus. Units differ in detail from thosein Collins et al. (2013).

\section{References:}

Pappalardo, R. T. et al. (2004) Geology of Ganymede, in Bagenal, F., ed., Jupiter. The Planet, Satellites and Magnetosphere, 363-396.

Collins, G. C. et al. (1998), Formation of Ganymede Grooved Terrain by Sequential Extensional Episodes: Implications of Galileo Observations for Regional Stratigraphy: Icarus, 135, 1, 345-359.

Patterson, G. W. et al. (2010) Global geological mapping of Ganymede: Icarus, 207, 2, 845-867.

Collins, G. C. et al. (2013) Global geologic map of Ganymede, p. 3237. 
Grasset, O. et al. (2013) JUpiter ICy moons Explorer (JUICE): An ESA mission to orbit Ganymede and to characterise the Jupiter system: PSS, 78, 1-21.

Neukum, G. et al. (1998). Cratering chronology in the Jovian system and the derivation of absolute ages: Lunar Planet. Sci. Conf. XXIX, abstr. No. 1742.

Zahnle, K. et al., (2003). Cratering rates in the outer Solar System: Icarus 163, 263-289. 